\title{
Common Fixed Points for Nonexpansive Type Mappings
}

\author{
VYOMESH PANT
}

\begin{abstract}
The aim of the present paper is to obtain common fixed point theorems for three mappings satisfying nonexpansive type condition. For this purpose we use the notion of pointwise $R$-weak commutativity or R-weak commutativity of type $\left(A_{g}\right)$ but without assuming the completeness of the space or continuity of the mappings involved. We further generalize the results obtained in first three theorems by replacing the condition of noncompatibility of maps with the property (E.A). In Theorem 5, we show that if the aspect of noncompatibility is taken in place of the property (E.A), the maps become discontinuous at their common fixed point. We are, thus, able to provide one more answer to the problem posed by Rhoades [13] regarding the existence of contractive definition which is strong enough to generate fixed point but does not forces the maps to become continuous. In Theorem 6 , we use the notion of conditionally commuting maps recently introduced by Pant and Pant [12] and prove a common fixed point theorem under minimal commutative condition.
\end{abstract}

\section{INTRODUCTION}

The study of common fixed points of compatible mappings emerged as an area of intense research activity ever since Jungck [2] introduced the notion of compatible mappings in 1986. However, the study of common fixed points of noncompatible mappings is also interesting. Work on these lines was initiated by Pant [5-8]. In the study of common fixed points of compatible mappings we often require assumptions on completeness of the space or continuity of the mappings involved besides some contractive condition, but the study of fixed points of noncompatible mappings can be extended to the class of nonexpansive or Lipschitz type mappings pairs $[7,11]$ even without assuming continuity of the mappings involved or completeness of the space.

Two selfmaps $f, g$ of a metric space $(X, d)$ are called $R$-weakly commuting (see Pant [5]) if there exists some real number $R>0$ such that

2000 Mathematics Subject Classification. Primary: 54H25.

Key words and phrases. Nonexpansive conditions, noncompatible mappings, Pointwise $R$-weak commutativity, $R$-weak commutativity, contractive conditions, property (E.A), Conditionally Commuting Maps. 
$d(f g x, g f x) \leq R(d(f x, g x))$ for all $x$ in $X . \quad f$ and $g$ are called pointwise $R$-weakly commuting if given $x$ in $X$, there exists $R>0$ such that $d(f g x, g f x) \leq R(d(f x, g x))$.

It was proved by Pant $[5,6,9]$ that pointwise $R$-weak commutativity is

(i) equivalent to commutativity at coincident points; and

(ii) a necessary, hence minimal, condition for the existence of common fixed points of contractive type mappings.

In 2006, Jungck and Rhoades [3] generalized the notion of $R$-weak commutativity by giving the notion of occasionally weak commutativity. Redefining the said notion of occasionally weak commutativity, in a recant paper, Pant and Pant [12] have introduced the concept of conditionally commuting maps. Two selfmappings $\mathrm{f}$ and $\mathrm{g}$ of a metric space $(X, d)$ are called conditionally commuting if they commute on a nonempty subset of the set of coincidence points whenever the set of their coincidences is nonempty.

Two selfmaps $f$ and $g$ of a metric space $(X, d)$ are called compatible (see Jungck [2]) if $\lim _{n} d\left(f g x_{n}, g f x_{n}\right)=0$, whenever $\left\{x_{n}\right\}$ is a sequence in $X$ such that $\lim _{n} f x_{n}=\lim _{n} g x_{n}=t$ for some $t$ in $X$. It is clear from the above definition that $f$ and $g$ will be noncompatible if there exists at least one sequence $\left\{x_{n}\right\}$ such that $\lim _{n} f x_{n}=\lim _{n} g x_{n}=t$ for some $t$ in $X$ but $\lim _{n} d\left(f g x_{n}, g f x_{n}\right)$ is either non-zero or non-existent. Compatibility implies pointwise $R$-weak commutativity since compatible maps commute at their coincidence points. However, as shown in the examples on the following pages, pointwise $R$-weakly commuting maps need not be compatible.

In 1997, Pathak et al [4] gave an analogue of $R$-weak commutativity by introducing the concept of $R$-weak commutativity of type $(\mathrm{Ag})$.

Two selfmappings $f$ and $g$ of a metric space $(X, d)$ are called $R$-weakly commuting of type $(\mathrm{Ag})$ (see [4]) if there exists some positive real number $R$ such that $d(f f x, g f x) \leq R d(f x, g x)$ for all $x$ in $X$.

In a recent work, Aamri and Moutawakil [1] introduced the property (E.A) and thus generalized the notion of noncompatible maps.

Let $f$ and $g$ be two selfmappings of a metric space $(X, d)$. We say that $f$ and $g$ satisfy the property (E.A) if there exists a sequence $\left\{x_{n}\right\}$ such that

$$
\lim _{n} f x_{n}=\lim _{n} g x_{n}=t
$$

for some $t$ in $X$. If two maps are noncompatible they satisfy the E.A property. The converse, however, is not necessarily true. To support our assertion, we quote examples from [1].

Example 1 ([1]). Let $X=[0,+\infty)$. Define $T, S: X \rightarrow X$ by

$$
T x=x / 4, \quad S x=3 x / 4, \quad \forall x \in X
$$

Consider the sequence $x_{n}=1 / n$. Clearly $\lim _{n} T x_{n}=\lim _{n} S x_{n}=0$. Then $T$ and $S$ satisfy E.A. 
Example 2 ([1]). Let $X=[2,+\infty)$. Define $T, S: X \rightarrow X$ by

$$
T x=x+1, \quad S x=2 x+1, \quad \forall x \in X .
$$

Suppose that property (E.A) holds; then there exists in $X$ a sequence $\left\{x_{n}\right\}$ satisfying $\lim _{n} T x_{n}=\lim _{n} S x_{n}=t$, for some $t \in X$.

Therefore $\lim _{n} x_{n}=t-1$ and $\lim _{n} x_{n}=(t-1) / 2$.

Then $t=1$, which is a contradiction since $1 \notin X$. Hence $T$ and $S$ do not satisfy E.A.

In the present paper, we first obtain common fixed point theorems for three mappings satisfying nonexpansive condition by employing the notion of pointwise $R$-weak commutativity and simple techniques of contraction maps (Theorem 1, Theorem 2 and Theorem 3). Theorem 4 is a common fixed point theorem for three mappings which are $R$-weakly commutative; wherein we replace the condition of noncompatibility with the property (E.A). All these theorems have been proved without assuming the completeness of the space or continuity of the mappings involved. In Theorem 5 we show that if the condition of noncompatibility is used in place of the property (E.A) the mappings become discontinuous at their common fixed points. Thus, we provide one more answer to the problem regarding the possibility of contractive definition which is strong enough to guarantee the existence of common fixed point but does not forces the maps to become continuous (Rhoades [13]). In Theorem 6 we further modify the results by using the notion of conditional commutativity.

\section{Results}

Theorem 1. Let $f, g$ and $h$ are selfmappings on a metric space $(X, d)$ satisfying the conditions

(i) $\overline{f X} \subset g X$, and $\overline{f X} \subset h X$, where $\overline{f X}$ denotes the closure of range of $f$

(ii) $d(f x, f y) \leq d(g x, h y)$, and

(iii) $d\left(f x, f^{2} y\right)<d(g x, h f y)$, whenever $f x \neq f^{2} y$.

Let $(f, g)$ and $(f, h)$ are pairs of pointwise $R$-weakly commuting mappings and either $(f, g)$ or $(f, h)$ be a pair of noncompatible mappings. Then $f, g$ and $h$ have a common fixed point.

Proof. Let $f$ and $g$ are noncompatible maps. Then there exists a sequence $\left\{x_{n}\right\}$ such that $\lim _{n} f x_{n}=\lim _{n} g x_{n}=t$ for some $t$ in $X$ but $\lim _{n} d\left(f g x_{n}, g f x_{n}\right)$ is either non-zero or non-existent. Since $\overline{f X} \subset h X$, for each $x_{n}$ there exists $y_{n}$ in $X$ such that $f x_{n}=h y_{n}$. Thus $f x_{n} \rightarrow t, g x_{n} \rightarrow t$ and $h y_{n} \rightarrow t$ as $n \rightarrow \infty$. By virtue of this and using (ii) we obtain that $\lim _{n} f y_{n} \rightarrow t$.

Since $t \in \overline{f X}$ and $\overline{f X} \subset h X$, there exists a point $u$ in $X$ such that $t=h u$. If $h u \neq f u$, the inequality

$$
d\left(f x_{n}, f u\right) \leq d\left(g x_{n}, h u\right)
$$


on letting $n \rightarrow \infty$ yields $f u=h u=t$. Since $\overline{f X} \subset g X$, there exists a point $w$ in $X$ such that $f u=g w$ and $f u=g w=h u=t$. If $f w \neq g w$, using (ii) again, the inequality

$$
d\left(f w, f y_{n}\right) \leq d\left(g w, h y_{n}\right)
$$

yields $f w=g w=t$ as $n \rightarrow \infty$.

Pointwise $R$-weak commutativity of $f$ and $g$ implies that $d(f g w, g f w) \leq$ $R_{1} d(f w, g w)=0$, for some $R_{1}>0$, that is, $f g w=g f w$ and $f f w=f g w=$ $g f w=g g w=h f w=h g w$. Pointwise $R$-weak commutativity of $f$ and $h$ implies that $d(f h u, h f u) \leq R_{2} d(f u, h u)=0$ for some $R_{2}>0$. Thus $f h u=h f u$ and $f f u=f h u=h f u=h h u$. Using (iii) we get

$$
d(f w, f f w)<d(g w, h f w)=d(f w, f f w),
$$

a contradiction. Hence, $f w=f f w=h f w$. Thus, $f w=f f w=g f w=h f w$ and, therefore, $f w$ is a common fixed point of $f, g$ and $h$. Hence the theorem is prooved.

We now give an example to illustrate the above theorem.

Example 3. Let $X=[2,6]$ and $d$ be the usual metric on $X$. Define $f, g$ and $h: X \rightarrow X$ as follows:

$$
\begin{gathered}
f x=\left\{\begin{array}{ll}
2, & 2 \leq x<3 \\
5, & x=3 \\
\frac{x+21}{12}, & 3<x \leq 6
\end{array} \quad g x= \begin{cases}2, & x=2 \\
6, & 2<x \leq 3 \\
x-1, & 3<x \leq 6\end{cases} \right. \\
h x= \begin{cases}x, & 2 \leq x<3 \\
5, & x \geq 3\end{cases}
\end{gathered}
$$

Then $f, g$ and $h$ satisfy all the conditions of the above theorem and have a unique common fixed point $x=2$. It may be verified in this example that $f X=[2,9 / 4] \cup 5, g X=[2,5] \cup 6, h X=[2,3) \cup 5$. Thus $\overline{f X} \subset g X$ and $\overline{f X} \subset$ $h X$. Also $f$ and $g$ are noncompatible pointwise $R$-weakly commuting maps. $f$ and $g$ are $R$-weakly commuting since they commute at their coincidence points. To see that $f$ and $g$ are noncompatible, let us consider the sequence $\left\{x_{n}=3+\frac{1}{n}, n \geqslant 1\right\}$. Then $f x_{n} \rightarrow 2, g x_{n} \rightarrow 2, f g x_{n}=2, g f x_{n}=6$. Hence $f$ and $g$ are noncompatible. It is also easy to verify that $f, g$ and $h$ satisfy the contractive condition $d(f x, f y) \leq d(g x, g y)$ together with the condition $d(f x, f f y)<d(g x, h f y)$.

Theorem 2. Let $f, g$ and $h$ be selfmappings on a metric space $(X, d)$ satisfying condition (i) and (ii) of Theorem 1 and

(iii) $d\left(f x, f^{2} y\right)>d(g x, h f y)$, whenever $f x \neq f^{2} y$.

Suppose $(f, g)$ and $(f, h)$ be pairs of pointwise $R$-weakly commuting maps and one of the pairs $(f, g)$ or $(f, h)$ is noncompatible. Then $f, g$ and $h$ have a common fixed point. 
The theorem can be proved in almost similar manner as in Theorem 1 .

As a corollary of Theorem 1 and Theorem 2 above, we find the following theorem.

Theorem 3. Let $f, g$ and $h$ be selfmappings on a metric space $(X, d)$ satisfying conditions

(i) $\overline{f X} \subset g X$, and $\overline{f X} \subset h X$, where $\overline{f X}$ denotes the closure of range of $f$

(ii) $d(f x, f y) \leq d(g x, h y)$, and

(iii) $d\left(f x, f^{2} y\right) \neq d(g x, h f y)$, whenever $f x \neq f^{2} y$.

Suppose $(f, g)$ and $(f, h)$ be pairs of pointwise $R$-weakly commuting maps and one of the pairs $(f, g)$ or $(f, h)$ is noncompatible. Then $f, g$ and h have a common fixed point.

In a recent work, Aamri and Moutawakil [1] introduced the property (E.A) and thus generalized the notion of noncompatible maps. Our next theorem is for $R$-weakly commutative maps of type $\left(A_{g}\right)$. We use the property (E.A) in place of noncompatibility.

Theorem 4. Let $f, g$ and $h$ be selfmappings on a metric space $(X, d)$ satisfying conditions

(i) $\overline{f X} \subset g X$, and $\overline{f X} \subset h X$, where $\overline{f X}$ denotes the closure of range of $f$

(ii) $d(f x, f y) \leq d(g x, h y)$, and

(iii) $d\left(f x, f^{2} y\right) \neq d(g x, h f y)$, whenever $f x \neq f^{2} y$.

Suppose $(f, g)$ and $(f, h)$ be pairs of pointwise $R$-weakly commuting maps and one of the pairs $(f, g)$ or $(f, h)$ satisfies the property (E.A). Then $f, g$ and $h$ have a common fixed point.

Proof. Since $f$ and $g$ satisfy the property (E.A), there exists a sequence $\left\{x_{n}\right\}$ such that $f x_{n} \rightarrow t$ and $g x_{n} \rightarrow t$ for some $t$ in $X$. Since $\overline{f X} \subset h X$, for each $x_{n}$ there exists $y_{n}$ in $X$ such that $f x_{n}=h y_{n}$. Thus $f x_{n} \rightarrow t, g x_{n} \rightarrow t$ and $h y_{n} \rightarrow t$ as $n \rightarrow \infty$. By virtue of this and using (ii) we obtain that $\lim _{n} f y_{n} \rightarrow t$.

Since $t \in \overline{f X}$ and $\overline{f X} \subset h X$, there exists a point $u$ in $X$ such that $t=h u$. If $h u \neq f u$, the inequality

$$
d\left(f x_{n}, f u\right) \leq d\left(g x_{n}, h u\right),
$$

yields $f u=h u=t$ when $n \rightarrow \infty$. Since $\overline{f X} \subset g X$, there exists a point $w$ in $X$ such that $f u=g w$ and $f u=g w=h u=t$. If $f w \neq g w$, using (ii) again, the inequality

$$
d\left(f w, f y_{n}\right) \leq d\left(g w, h y_{n}\right),
$$

yields $f w=g w=t$ as $n \rightarrow \infty$.

Pointwise $R$-weak commutativity of $f$ and $g$ implies that $d(f g w, g f w) \leq$ $R_{1} d(f w, g w)=0$, for some $R_{1}>0$, that is, $f g w=g f w$ and $f f w=f g w=$ 
$g f w=g g w=h f w=h g w$. Pointwise $R$-weak commutativity of $f$ and $h$ implies that $d(f h u, h f u) \leq R_{2} d(f u, h u)=0$ for some $R_{2}>0$. Thus $f h u=h f u$ and $f f u=f h u=h f u=h h u$. Using (iii) we get

$$
d(f w, f f w) \neq d(g w, h f w)=d(f w, f f w),
$$

a contradiction. Hence, $f w=f f w=h f w$. Thus, $f w=f f w=g f w=h f w$ and, therefore, $f w$ is a common fixed point of $f, g$ and $h$. Hence the theorem is proved.

In the next theorem, we show that if we use the notion of noncompatibility in place of the property (E.A), the mappings become discontinuous at their common fixed point. Thus, we provide one more answer to the problem regarding the existence of contractive definition which is strong enough to guarantee the existence of common fixed point but does not forces the maps to become continuous (Rhoades [13]).

Theorem 5. Let $f, g$ and $h$ be selfmappings on a metric space $(X, d)$ satisfying conditions

(i) $\overline{f X} \subset g X$, and $\overline{f X} \subset h X$, where $\overline{f X}$ denotes the closure of range of $f$

(ii) $d(f x, f y) \leq d(g x, h y)$, and

iii $d\left(f x, f^{2} y\right) \neq d(g x, h f y)$, whenever $f x \neq f^{2} y$.

Suppose $(f, g)$ and $(f, h)$ be pairs of pointwise $R$-weakly commuting maps of type $\left(A_{g}\right)$ and one of the pairs $(f, g)$ or $(f, h)$ is noncompatible. Then $f, g$ and $h$ have a common fixed point and the fixed point is a point of discontinuity.

Proof. Let $f$ and $g$ are noncompatible maps. Then there exists a sequence $\left\{x_{n}\right\}$ such that

$$
\lim _{n} f x_{n}=\lim _{n} g x_{n}=t \quad \text { for some } t \text { in } X,
$$

but $\lim _{n} d\left(f g x_{n}, g f x_{n}\right)$ is either non-zero or non-existent. Since $\overline{f X} \subset h X$, for each $x_{n}$ there exists $y_{n}$ in $X$ such that $f x_{n}=h y_{n}$. Thus $f x_{n} \rightarrow t$, $g x_{n} \rightarrow t$ and $h y_{n} \rightarrow t$ and as $n \rightarrow \infty$. By virtue of this and using (ii) we obtain that $\lim _{n} f y_{n} \rightarrow t$.

Since $t \in \overline{f X}$ and $\overline{f X} \subset h X$, there exists a point $u$ in $X$ such that $t=h u$. If $h u \neq f u$, the inequality

$$
d\left(f x_{n}, f u\right) \leq d\left(g x_{n}, h u\right),
$$

which on letting $n \rightarrow \infty$ yields $f u=h u=t$. Since $\overline{f X} \subset g X$, there exists a point $w$ in $X$ such that $f u=g w$ and $f u=g w=h u=t$. If $f w \neq g w$, using (ii) again, the inequality

$$
d\left(f w, f y_{n}\right) \leq d\left(g w, h y_{n}\right),
$$

yields $f w=g w=t$ as $n \rightarrow \infty$. 
Pointwise $R$-weak commutativity of $f$ and $g$ implies that $d(f g w, g f w) \leq$ $R_{1} d(f w, g w)=0$, for some $R_{1}>0$, that is, $f g w=g f w$ and $f f w=f g w=$ $g f w=g g w=h f w=h g w$. Pointwise $R$-weak commutativity of $f$ and $h$ implies that $d(f h u, h f u) \leq R_{2} d(f u, h u)=0$ for some $R_{2}>0$. Thus $f h u=h f u$ and $f f u=f h u=h f u=h h u$. Using (iii) we get

$$
d(f w, f f w) \neq d(g w, h f w)=d(f w, f f w),
$$

a contradiction. Hence, $f w=f f w=h f w$. Thus, $f w=f f w=g f w=h f w$ and, therefore, $f w$ is a common fixed point of $f, g$ and $h$. Hence the first part is proved.

We now show that $f, g$ and $h$ are discontinuous at the common fixed point. First we suppose that $f$ and $g$ are noncompatible pair of mappings and show that $f$ and $g$ are discontinuous at the common fixed point $t=f u=g u$. If possible, suppose $f$ is continuous. Then considering the sequence $\left\{x_{n}\right\}$ as assumed above, we get $\lim _{n} f f x_{n}=f t=t . R$-weak commutativity of type $\left(A_{g}\right)$ implies that $d\left(f f x_{n}, g f x_{n}\right) \leq R d\left(f x_{n}, g x_{n}\right)$. On letting $\rightarrow \infty$ this yields $\lim _{n} g f x_{n}=f t=t$. This, in turn, yields $\lim _{n} d\left(f g x_{n}, g f x_{n}\right)=$ $d(f t, f t)=0$. This contradicts the fact that $\lim _{n} d\left(f g x_{n}, g f x_{n}\right)$ is either nonzero or nonexistent for the sequence $\left\{x_{n}\right\}$ of (1). Hence $f$ is discontinuous at the fixed point. Next, suppose that $g$ is continuous. Then for the sequence $\left\{x_{n}\right\}$ of (1), we get $\lim _{n} g f x_{n}=g t=t$ and $\lim _{n} g g x_{n}=g t=t$. In view of these limits, the inequality

$$
d\left(f t, f g x_{n}\right) \neq d\left(g t, h f x_{n}\right)
$$

yields a contradiction unless $\lim _{n} f g x_{n}=f t=g t$. But $\lim _{n} f g x_{n}=g t$ and $\lim _{n} g f x_{n}=g t$ contradicts the fact that $\lim _{n} d\left(f g x_{n}, g f x_{n}\right)$ is either nonzero or nonexistent. Thus both $f$ and $g$ are discontinuous at their common fixed point. Similarly it can be shown that $f$ and $h$ are discontinuous at the common fixed point. Hence the theorem is proved.

Remark 1. In a recent work, Pant and Pant [12] have generalized the notion of $R$-weak commutativity by defining the concept of conditional commutativity. Preceding Theorems can be generalized if the notion of $R$-weak commutativity is replaced by the notion of conditional commutativity. We now prove a theorem under the assumption of conditional commutativity.

Theorem 6. Let $f, g$ and $h$ be selfmappings on a metric space $(X, d)$ satisfying conditions

(i) $\overline{f X} \subset g X$, and $\overline{f X} \subset h X$, where $\overline{f X}$ denotes the closure of range of $f$

(ii) $d(f x, f y) \leq d(g x, h y)$, and

(iii) $d\left(f x, f^{2} y\right) \neq d(g x, h f y)$, whenever $f x \neq f^{2} y$.

Suppose $(f, g)$ and $(f, h)$ be pairs of conditionally commuting maps and one of the pairs $(f, g)$ or $(f, h)$ satisfies the property (E.A). Then $f, g$ and $h$ have a common fixed point. 
Proof. Since $f$ and $g$ satisfy the property (E.A), there exists a sequence $\left\{x_{n}\right\}$ such that $f x_{n} \rightarrow t$ and $g x_{n} \rightarrow t$ for some $t$ in $X$. Since $\overline{f X} \subset h X$, for each $x_{n}$ there exists $y_{n}$ in $X$ such that $f x_{n}=h y_{n}$. Thus $f x_{n} \rightarrow t, g x_{n} \rightarrow t$ and $h y_{n} \rightarrow t$ as $n \rightarrow \infty$. By virtue of this and using (ii) we obtain that $\lim _{n} f y_{n} \rightarrow t$.

Since $t \in \overline{f X}$ and $\overline{f X} \subset h X$, there exists a point $u$ in $X$ such that $t=h u$. If $h u \neq f u$, the inequality

$$
d\left(f x_{n}, f u\right) \leq d\left(g x_{n}, h u\right),
$$

which on letting $n \rightarrow \infty$ yields $f u=h u=t$. Since $\overline{f X} \subset g X$, there exists a point $w$ in $X$ such that $f u=g w$ and $f u=g w=h u=t$. If $f \neq g w$, using (ii) again, the inequality

$$
d\left(f w, f y_{n}\right) \leq d\left(g w, h y_{n}\right),
$$

yields $f w=g w=t$ as $n \rightarrow \infty$.

Since $f$ and $g$ are conditionally commuting, two cases arise: $f$ and $g$ may or may not commute at $w$. If $f$ and $g$ commute at $w, f g w=g f w$. Also, $f f w=f g w=g f w=g g w$. We claim that $f f w=f w$. If not by virtue of (iii) we get

$$
d(f f w, f u)<d(g f w, h u)=d(f f w, f u),
$$

a contradiction. Hence, $f w=f f w=g f w$. Hence $f w$ is a common fixed point of $f$ and $g$. If $f w \neq h f w$, then the inequality $d(f w, f f w) \neq$ $d(g w, h f w)$ yields a contradiction unless $f w=f f w=h f w$. Hence $f w$ is a common fixed point of $f$ and $h$. Thus $f w$ is a common fixed point of $f, g$ and $h$.

If $f$ and $g$ do not commute at $w$, then by virtue of conditional commutativity of $f$ and $g$, there exists a coincidence point of $f$ and $g$ at which $f$ and $g$ commute, that is, there exists a point $v$ in $X$ such that $f v=g v$ and $f g v=g f v$. Rest of the proof can be completed on the similar lines as has been done in the case when $f$ and $g$ commute at $w$.

\section{REFERENCES}

[1] M. Aamri and D.El Moutawakil, Some New Common Fixed Point Theorems Under Strict Contractive Conditions, J. Math. Anal. Appl., 270 (2002), 181-188.

[2] G. Jungck, Compatible Mappings And Common Fixed Points, Internat. J. Math. Math. Sci., 9 (1986), 771-779.

[3] G. Jungck and B.E. Rhoades, Fixed Point Theorems for Occasionally Weakly Compatible Mappings, Fixed Point Theory, 7-2 (2006), 287-296.

[4] H.K. Pathak, Y.J. Cho and S.M. Kang, Remarks on R-Weakly Commuting Mappings and Common Fixed Point Theorems, Bull. Korean Math. Soc., 34 (1997), 247-257.

[5] R.P. Pant, Common fixed points of noncommuting mappings, J. Math. Anal. Appl., 188 (1994), 436-440. 
[6] R.P. Pant, R-weak commutativity and common fixed points of noncompatible maps, Ganita, 49 (1998), 19-27.

[7] R.P. Pant, Common fixed point theorems for contractive maps, J. Math. Anal. Appl., 226 (1998), 251-258.

[8] R.P. Pant, Common fixed points of Lipschitz type mapping pairs, J. Math. Anal. Appl., 240 (1999), 280-283.

[9] R.P. Pant, Discontinuity and fixed points, J. Math. Anal. Appl., 240 (1999), 284-289.

[10] R.P. Pant, R-weak Commutativity and common fixed points, Soochow J. Math., 25 (1999), 37-42.

[11] R.P. Pant and Vyomesh Pant, Lipschitz Type Condition and Common Fixed Points, J. Indian Math Soc., 67 (2000), 179-182.

[12] Vyomesh Pant and R.P. Pant, Common Fixed Points of Conditionally Commuting Maps, Fixed Point Theory, 11 (2010)(1), 113-118.

[13] B.E. Rhoades, Contractive Definitions and Continuity, Contemporary Math., 72 (1988), 233-245.

VYOMESH PANT

A -24, J.K. PURAM

Choti Mukhani, Haldwani - 263139

NAINITAL, UTTARAKHAND

INDIA

E-mail address: vyomeshpant@yahoo.co.in 
